\title{
HARQ com combinação de pacotes em Sistemas IEEE 802.11a
}

\author{
André Noll Barreto
}

\begin{abstract}
Resumo-Na tecnologia de redes locais sem fio IEEE 802.11a é previsto o uso de ARQ para a retransmissão de pacotes recebidos erroneamente. É entretanto sabido que a combinação de ARQ com técnicas de codificação com correção de erros, também conhecida como ARQ Híbrido (HARQ) pode trazer ganhos substanciais de desempenho a sistemas de transmissão de pacotes. Mostramos neste artigo que, levando-se em conta algumas restrições impostas pela norma, o uso de HARQ com combinação de pacotes tipo Chase também é possível em sistemas 802.11a, apenas com algumas alterações no receptor, e que ganhos consideráveis de desempenho podem ser obtidos desta maneira.
\end{abstract}

Palavras-Chave - combinação de pacotes, HARQ, redes locais sem fio, IEEE 802.16a

Abstract-IEEE 802.11a-based WLANs make use of HARQ for retransmission of packets received with error. It is however widely known that Hybrid ARQ (HARQ), which is the combination of ARQ with error-correcting codes may bring about substantial performance gains for packet transmission systems. We show in this paper that the use of HARQ with Chase combining is also possible in 802.11a systems, even taking into account some restrictions imposed by the standards. Our investigations show that considerable throughput gains are achievable with only some changes to the reception structure.

Keywords-Chase combining, HARQ, WLAN, IEEE 802.16a

\section{Introdução}

Redes locais sem fio (WLAN - Wireless Local Area Networks) baseadas na família de normas IEEE 802.11 se popularizaram bastante nos últimos anos, por causa de seus baixos custo e facilidade de instalação, e encontram diversas aplicações nos ambientes residencial e corporativo, assim como para acesso público em áreas de alta demanda de tráfego de dados, como aeroportos, centros empresariais, hotéis, etc.

Após o sucesso inicial de redes baseadas no sistema IEEE $802.11 \mathrm{~b}$, baseado em espalhamento espectral, que opera na faixa não licenciada de $2,4 \mathrm{GHz}$ com taxa de dados de até $11 \mathrm{Mbps}$, foram criadas as normas IEEE 802.11a e IEEE $802.11 \mathrm{~g}$, operando nas faixas de $5 \mathrm{GHz}$ e $2,4 \mathrm{GHz}$ respectivamente, mas ambas utilizando OFDM (Orthogonal Frequency Division Multiple Access) para permitir a transmissão de taxas de até $54 \mathrm{Mbps}$ em um canal de $25 \mathrm{MHz}$ em um ambiente com seletividade na frequiência [1]-[2].

Os sistemas 802.11 de redes locais sem fio são baseados na transmissão de pacotes, e dependem de técnicas como códigos corretores de erro, verificação de redundância cíclica (CRC - Cyclic Redundancy Check) e repetição de pacotes via ARQ (Automatic Repeat request) para garantir a transmissão confiável de pacotes de dados em um ambiente sem fio hostil.

André Noll Barreto, Instituto Nokia de Tecnologia (INdT), Brasília - DF, e-mail: andre.barreto@indt.org.br

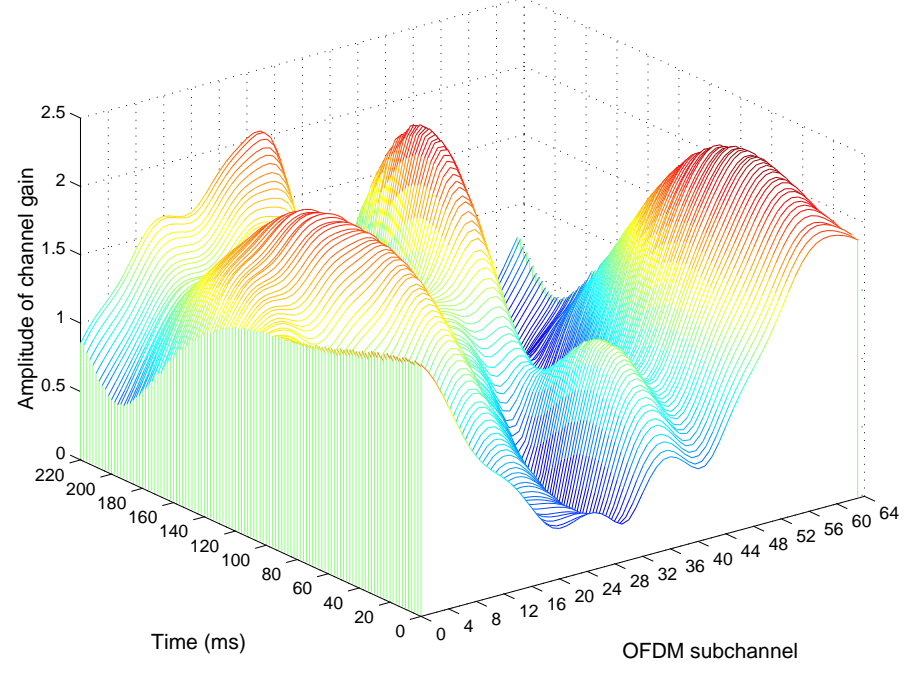

Fig. 1. Exemplo típico de canal no tempo e na freqüência

Redes WLAN banda larga têm como alvo usuários estáticos ou com baixa mobilidade em um ambiente indoor, onde tipicamente o canal varia muito lentamente. Por exemplo, para um usuário caminhando com velocidade $V \simeq 1 \mathrm{~m} / \mathrm{s}$ e freqüência de portadora $f_{c}=5 \mathrm{GHz}$ o espalhamento de Doppler máximo é $f_{d}=V f_{c} / c=16.67 \mathrm{~Hz}$, o que nos dá um intervalo de coerência $T_{c} \simeq 1 / 2 f_{d}=30 \mathrm{~ms}$. Nestas condições, se o enlace estiver num período de desvanecimento severo, apenas com ARQ o usuário pode retransmitir diversas vezes o mesmo pacote, que não conseguirá ser detectado sem erros. Um exemplo de um canal típico pode ser visto na Fig. 1, onde podemos ver que desvanecimentos profundos podem ter duração da ordem de dezenas de milisegundos.

Uma solução para este problema é o uso de ARQ Híbrido (HARQ), que é a combinação de retransmissão de pacotes (ARQ) com esquemas de codificação corretora de erros. HARQ está padronizado em diversas tecnologias, como por exemplo no EDGE, ou no WiMAX. Existem diferentes algoritmos de HARQ, sendo o mais simples o HARQ Tipo I [3], descrito a seguir.

Uma melhora significativa no desempenho de transmissão pode ser alcançada se os pacotes retransmitidos forem combinados antes da decodificação. Mesmo pacotes com erro contêm alguma informação útil, sendo um desperdício descartá-los. Em vez disso, podemos armazenar os valores recebidos e combiná-los com o sinal recebido nas retransmissões subseqüentes. Este método, conhecido como combinação de código ou de pacotes, ou combinação de Chase [6], já foi extensivamente estudado na literatura, por exemplo em [5]-[10]. Foi mostrado 
nestes estudos que através da combinação de pacotes é possível a transmissão de pacotes mesmo em condições muito desfavoráveis de razão sinal-interferência/ruído (SINR), o que não é possível apenas com ARQ.

Em [11] foi estudada a implementação em um sistema IEEE 802.11a de um HARQ Tipo II com redundância incremental, o que requer entretanto modificações substanciais nas normas existentes. Também não foram investigados em [11] aspectos práticos da implementação de HARQ no IEEE 802.11a, como a existência de seqüências de espalhamento variáveis e a impossibilidade de se aplicar HARQ no cabeçalho do pacote. Estes mesmos aspectos também não foram considerados em [12], onde um esquema de HARQ necessitando de um canal de retorno é sugerido, o que também exigiria modificações significativas nos padrões. De qualquer modo, estes trabalhos mostraram que HARQ pode ser bastante efetivo em aumentar a vazão na transmissão de dados em redes WLAN, e veremos que, diferentemente de outros esquemas de HARQ como redundância incremental, combinação de pacotes pode ser implementada em sistemas IEEE 802.11a sem modificações nos padrões existentes. A camada física do IEEE802.11g é muito similar ao IEEE802.a, e a mesma análise se aplica. Neste artigo examinaremos como pode ser feita esta implementação, em face de algumas restrições impostas pela norma vigente, e veremos os ganhos de desempenho que podem ser atingidos com combinação de pacotes. Faremos também uma sugestão de como combinação de pacotes pode ser melhor aproveitada com uma pequena modificação na norma.

Na Seção II fazemos uma descrição matemática do sistema considerado e na Seção III descrevemos a implementação da combinação de pacotes. Finalmente na Seção IV apresentamos alguns resultados de simulação.

\section{DESCRIÇÃo DO Sistema}

Nesta seção descrevemos matematicamente a transmissão de dados em um sistema OFDM, como o padronizado na norma IEEE 802.11a de redes locais sem fio. Na seguinte descrição, as letras em negrito representam vetores de dados. No transmissor são gerados pacotes de dados na camada MAC, de até 2312 bytes, e cada pacote é enviado em um quadro de transmissão. Supomos na entrada um pacote com $N_{\mathrm{p}}$ bits $d(n)$, que é codificado com um código convolucional de taxa $R_{c}$, gerando $N_{c}=N_{p} / R_{c}$ bits de codificação $\mathbf{b}_{i}$. Estes bits são entrelaçados e mapeados em $N_{s}=N_{c} / \log _{2}(M)$ símbolos QAM ou QPSK, onde $M$ é o tamanho da constelação. Esta seqüência de símbolos é dividida em blocos $\mathbf{x}_{i}$ de $K_{s}$ símbolos, onde cada símbolo é transmitido em uma subportadora diferente. São introduzidas $K_{p}$ subportadoras piloto para estimação de canal e sincronização e $K_{z}$ subportadoras nulas como banda de guarda e na subportadora DC. Cada bloco assim formado passa por uma transformada de Fourier inversa rápida (IFFT) de tamanho $K$, com $K=K_{d}+K_{p}+K_{z}$. A saída da IFFT corresponde ao sinal amostrado no tempo, com intervalo entre amostras $T_{a}=1 /(\Delta f K)$, onde $\Delta f$ é o espaçamento entre subportadoras. A cada bloco de $K$ amostras na saída da IFFT é adicionado um prefixo cíclico de $G$ amostras, com duração $T_{G}=G T_{a}$. Este prefixo cíclico possibilita a eliminação de interferência entre símbolos causada por multipercursos com um atraso de até $T_{G}$. Estas $K+G$ amostras correspondem a um símbolo OFDM, que tem portanto uma duração $T_{t}=(K+G) T_{a}$, na qual são transmitidos $K_{b}=K_{d} \log _{2}(M) R_{c}$ bits. A seqüência de símbolos OFDM passa por um filtro de transmissão, e este sinal é então convertido para rádio-frequiência e transmitido por um canal com múltiplos percursos e desvanecimento.

No IEEE 802.11a o espaçamento entre subportadoras $\Delta f=$ $312,5 k H z$, e o tamanho da IFFT é $K=64$, o que resulta numa freqüência de amostragem $f_{a}=1 / T_{a}=20 \mathrm{MHz}$. O intervalo de guarda consiste de 16 amostras, o que nos dá um símbolo OFDM de duração $T_{t}=4 \mathrm{~ms}$. Das 64 subportadoras, $K_{d}=48$ são utilizadas para dados e $K_{p}=4$ são canais piloto. O IEEE 802.11a permite o uso de 8 diferentes esquemas de codificação e modulação, com taxas de dados entre 6 e 54 Mbps. Estas diferentes taxas são alcançadas com combinações diferentes das modulações BPSK, QPSK, 16-QAM e 64QAM, e com com um código convolucional de taxa $R_{c}=1 / 2$, com taxas maiores $\left(R_{c}=2 / 3\right.$ e $\left.R_{c}=3 / 4\right)$ podendo ser alcançadas por meio de puncturing.

No receptor são realizadas as operações inversas àquelas do transmissor. $\mathrm{O}$ sinal recebido passa por um filtro de recepção, é convertido para banda básica e amostrado a uma taxa $1 / T_{a}$. A extensão cíclica é removida e uma transformada rápida de Fourier (FFT) é realizada. As subportadoras nulas e piloto são removidas, e o sinal recebido no $k$-ésimo subcanal e no $i$-ésimo símbolo OFDM após esta operação é

$$
r_{i, k}=h_{k} x_{i, k}+\nu_{i, k},
$$

onde $h_{k}$ é o ganho do $k$-ésimo subcanal e $\nu_{i, k}$ é o componente de ruído branco gaussiano complexo com variância $\sigma^{2}=N_{0}$.

Baseado nas estimativas de canal $\hat{h}_{k}$, podemos equalizar o sinal recebido para obter

$$
y_{i, k}=\frac{r_{i, k}}{\hat{h}_{k}} .
$$

Supondo que estimativas de canal perfeitas estão disponíveis, temos

$$
y_{i, k}=x_{i, k}+\nu_{i, k}^{\prime},
$$

onde $\nu_{i, k}^{\prime}$ é o componente de ruído branco gaussiano complexo com variância

$$
\sigma_{k}^{\prime 2}=N_{0} /\left|h_{k}\right|^{2}
$$

Consideramos no receptor um decodificador de Viterbi com entrada soft. Para simplificar a análise, suponhamos o uso de modulação BPSK, ou seja $x_{i, k}=2\left(b_{i, k}-1\right)$. A entrada do decodificador de Viterbi é o logaritmo da razão de verossimilhança (LLR - log-likelihhod ratio)

$$
\Lambda_{i, k}=\log \frac{\operatorname{Pr}\left\{y_{i, k} \mid b_{i, k}=1\right\}}{\operatorname{Pr}\left\{y_{i, k} \mid b_{i, k}=0\right\}},
$$

que, como $\nu_{k}$ tem uma distribuição gaussiana, é dada por 


$$
\Lambda_{i, k}=\frac{2}{\sigma^{2}} y_{i, k}\left|h_{k}\right|^{2}=\frac{2}{\sigma^{2}} r_{i, k} h_{k}^{*}
$$

Partindo do princípio que $\sigma^{2}$ é igual para todas as subportadoras e para todos os símbolos, o termo multiplicativo $2 / \sigma^{2}$ pode ser ignorado pelo algoritmo de Viterbi.

\section{HARQ TIPo I - COMBINAÇÃo DE PACOTES}

Agora suponhamos que a mesma informação é transmitida em $L$ pacotes diferentes. Isto pode ser visto como uma transmissão apenas, com um código de taxa $R_{c}^{\prime}=R_{c} / L$, composto da concatenação do código original de taxa $R_{c}$ e um código de repetição. Como mostrado em [6] este código equivalente tem propriedades de distância apenas levemente mais desfavoráveis que aquelas de um código de máxima distância livre de mesma taxa $R_{c}^{\prime}$. A essência deste esquema de combinação de códigos é que, com a diminuição da taxa de codificação, a mesma informação pode ser transmitida confiavelmente a uma razão sinal ruído (RSR) mais baixa. As $L$ versões repetidas de um bit de código $y_{i, k, l}$, com $0 \leq l<L$, pertencem ao mesmo ramo do decodificador de Viterbi, e precisamos agora obter a razão de verossimilhança

$$
\begin{aligned}
\Lambda_{i, k}^{L} & =\log \frac{\operatorname{Pr}\left\{y_{i, k, 0}, y_{i, k, 1}, \ldots, y_{i, k, L-1} \mid b_{i, k}=1\right\}}{\operatorname{Pr}\left\{y_{i, k, 0}, y_{i, k, 1}, \ldots, y_{i, k, L-1} \mid b_{i, k}=0\right\}} \\
& =\log \prod_{l=0}^{L-1} \frac{\operatorname{Pr}\left\{y_{i, k, l} \mid b_{i, k}=1\right\}}{\operatorname{Pr}\left\{y_{i, k, l} \mid b_{i, k}=0\right\}} \\
& =\sum_{l=0}^{L-1} \Lambda_{i, k, l}
\end{aligned}
$$

com

$$
\Lambda_{i, k, l}=r_{i, k, l} h_{k, l}^{*} .
$$

Pelas equações acima podemos ver que combinação de código é equivalente à combinação de razão máxima (MRC - Maximal Ratio Combining). Expressões similares podem ser obtidos para QPSK ou QAM com codificação de Gray, exceto que agora cada símbolo $x_{k}$ corresponde a vários bits de código, e, no caso de QAM, expressões diferentes para o cálculo da LLR podem ser necessárias para diferentes bits de código.

\section{Implementação em IEEE 802.11 a}

No IEEE 802.11 o esquema de ARQ consiste simplesmente na retransmissão de um pacote se nenhuma confirmação de recebimento $(\mathrm{ACK})$ for recebida dentro de um certo período, o que torna a implementação de repetição pacotes (HARQ Tipo I) simples em termos de protocolo. Entretanto, alguns aspectos específicos da camada física devem ser levados em conta na implementação.

Inicialmente precisamos levar em conta que os bits de informação são embaralhados antes da codificação, de modo que pacotes repetidos não são exatamente os mesmos. Suponhamos que d é a seqüência de bits da informação desejada. Na l-ésima retransmissão do pacote a seqüência de embaralhamento $\mathbf{c}_{l}$ é empregada, e a sequiência de dados

$$
\mathbf{d}_{l}=\mathbf{c}_{l} \oplus \mathbf{d}
$$

é transmitida, onde $\oplus$ é o operador de módulo de adição binária.

Agora, façamos $f_{c}(\cdot)$ representar a operação de codificação. Já que códigos convolucionais lineares são empregados, temos

$$
\mathbf{b}_{l}=f_{c}\left(\mathbf{d}_{l}\right)=f_{c}\left(\mathbf{c}_{l}\right) \oplus f_{c}(\mathbf{d}) .
$$

Portanto, o embaralhamento pode ser levado em conta no receptor mudando (7) para

$$
\Lambda_{i, k}^{L}=\sum_{l=0}^{L-1}\left(2 c_{i, k, l}-1\right) \Lambda_{i, k, l},
$$

onde $c_{i, k, l}$ corresponde ao $k$-ésimo subcanal do $i$-ésimo símbolo da $l$-ésima seqüência de espalhamento codificada.

Um dos aspectos críticos no uso de HARQ é a necessidade de o transmissor armazenar os valores recebidos de cada retransmissão mal sucedida. Como já mencionado, o esquema de combinação de códigos ótimo corresponde a combinação de razão máxima do sinal recebido $r_{i, k, l}$. Podemos portanto armazenar os valores equalizados do sinal recebido ou a razão de verossimilhança de cada bit de código. Ambas as opções são iguais no caso de modulação BPSK, mas para modulações de ordem mais alta cada símbolo corresponde a vários códigos de bit. A primeira opção, armazenar os valores recebidos $y_{i, k}$ tem como vantagem necessitar de menos memória. Entretanto, como a norma não prevê a sinalização do uso de HARQ, o transmissor pode retransmitir o pacote utilizando um outro esquema de modulação e codificação. Neste caso é necessário a armazenagem dos valores LLR $\Lambda_{i, k, l}$, o que torna o algoritmo de combinação independente do esquema de modulação. Diferentes taxas de codificação também podem ser consideradas sem dificuldades, já que todos os esquemas de codificação se baseiam no puncturing de um mesmo código base.

A seqüência de embaralhamento é obtida a partir dos sete primeiro bits do campo SERVICE de 16 bits, que é transmitido no princípio de cada pacote. Isto significa que temos antes que detetar estes bits sem empregar combinação de pacotes. Além disso, o cabeçalho MAC é transmitido no começo de cada pacote. Dentre outras coisas, este cabeçalho contém o endereço de destino do pacote e um número de sequiência de pacote, e combinação de pacotes só deve ser aplicada se estes parâmetros forem os mesmos que para os pacotes armazenados. Conseqüentemente, o cabeçalho MAC também deve ser detetado sem empregar combinação de pacotes. O cabeçalho MAC tem tipicamente 24 bytes em um pacote de dados, e ele pode ser diferente a cada retransmissão de um mesmo pacote, por exemplo no bit de retentativa, que é zero na primeira transmissão de um pacote e um em caso de retransmissão. Portanto, também os quatro últimos bytes do pacote, que correspondem à seqüência de verificação de quadro (FCS - Frame Check Sequence), assim como os seis bits de terminação do código convolucional, podem ser diferentes a cada retransmissão, e não podem ser combinados.

Como resultado das considerações acima, temos que combinação de pacotes só pode ser aplicada nos bits de dados, 
mas não nos bits de overhead. Podemos entretanto esperar que o comprimento dos pacotes seja da ordem de grandeza de centenas de bytes, até o máximo de 2312 bytes, de modo que a perda de overhead seja mantida pequena. O overhead total é de cerca de 31 bytes. Se a RSR não for muito baixa, um pacote com erro contém usualmente apenas alguns poucos bits errados, que têm uma probabilidade maior de ocorrerem entre os bits de dados, que são mais numerosos. Tendo isto em mente podemos ver que combinação de pacotes mantém grande parte de sua eficiência, mesmo quando aplicada apenas aos bits de dados.

Para a combinação de pacotes a camada física deve ter algum conhecimento sobre a camada MAC acima. Em particular, o tamanho do cabeçalho e do FCS, e a posição do número de seqüência e do endereço dentro do cabeçalho têm que ser conhecidos. Isto é em princípio conflitante com o modelo de camadas ISO/OSI, mas não requer modificações na norma. Podemos dizer portanto que esta técnica é uma espécie de algoritmo através de camadas (cross-layer). Além disso, combinação de pacotes é transparente para a camada MAC, que não precisa saber se combinação de pacotes é empregada. A decisão sobre as retransmissões permanece sob controle da camada MAC, com única diferença que os pacotes recebidos pela camada física têm uma probabilidade menor de apresentarem erros após algumas poucas retransmissões. Entretanto, o armazenamento dos valores recebidos para os diferentes pacotes pode ser mais eficiente se a camada física for informada pela camada MAC se o pacote foi recebido corretamente, o que é decidido baseado nos bits FCS. Desta maneira a camada física não tem que armazenar todos os pacotes, mas apenas aqueles sabidamente com erros.

\section{Resultados De Desempenho}

Nesta seção investigamos o desempenho de um sistema IEEE 802.11a empregando combinação de pacotes por meio de simulações de enlace. Utilizamos um simulador desenvolvido em MATLAB. Consideramos a transmissão em um canal com desvanecimento Rayleigh seletivo na freqüência, mais especificamente o modelo de canal ETSI tipo A para pequenos escritórios [13]. Consideramos também um esquema de retransmissão simples, no qual pacotes errôneos são simplesmente retransmitidos no próximo quadro possível. O canal é considerado constante até a recepção correta do pacote, o que é razoável num ambiente quase estático, como o esperado para aplicações de banda larga sem fio, desde que não sejam necessárias muitas retransmissões. Nas nossas simulações limitamos o número de retransmissões para dez, após o que o pacote é considerado como perdido.

Na Fig. 2 a vazão (throughput) com e sem combinação de pacotes é mostrada com diferentes comprimentos de pacotes e diferentes taxas de dados (diferentes esquemas de modulação e codificação), em função da RSR, representada pela razão energia de símbolo por densidade espectral de potência do ruído $E_{s} / N_{0}$. A vazão é definida aqui como o número de pacotes recebidos corretamente dividida pelo total de pacotes transmitidos. Podemos ver que o desempenho melhora pouco com combinação de códigos para pacotes

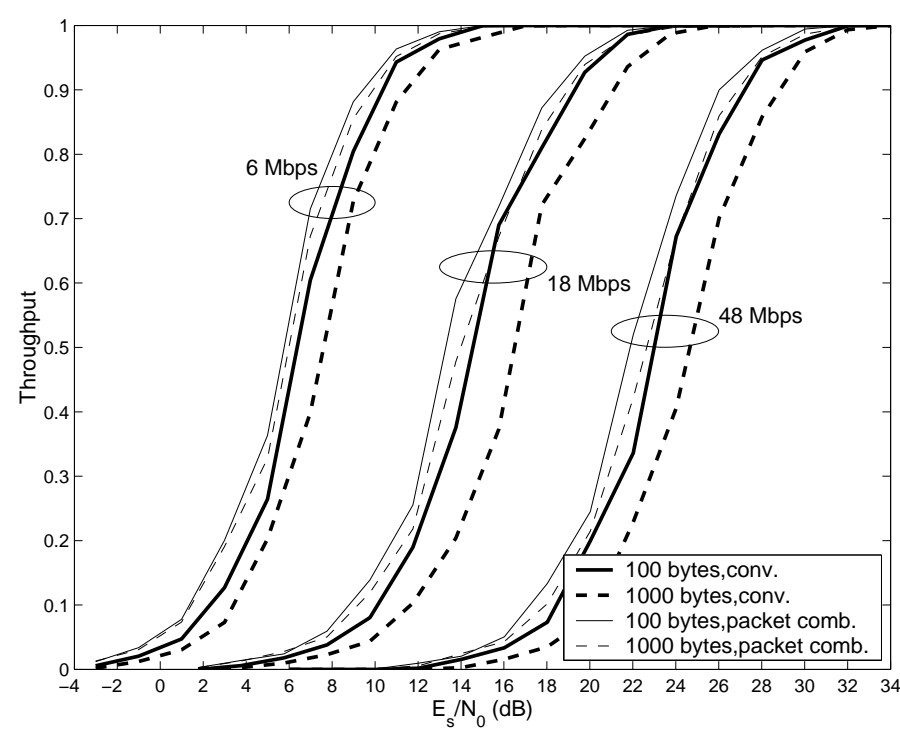

Fig. 2. Vazão com combinação de pacotes e diferentes tamanhos de pacotes

curtos, porque neste caso uma grande parte dos erros de bits acontecem no cabeçalho, que, como já vimos, não pode ser combinado. Pacotes mais longos têm uma taxa de erro de pacotes (PER) mais alta, e portanto uma vazão mais baixa, mas o ganho de desempenho relativo é maior com o emprego de combinação e pacotes. Isto ocorre porque, como mencionado na Seção III, para baixas taxas de erro a maioria dos erros ocorre com grande probabilidade na seqüência de dados, cuja confiabilidade pode ser aumentada através de combinação de pacotes.

Na Fig. 3 podemos ver a taxa de erro de pacotes com diferentes números de retransmissões, com e sem combinação de pacotes. Vemos que sem combinação a taxa de erros de pacotes pouco melhora com o aumento do número de retransmissões, enquanto que com combinação cada retransmissão traz uma melhora significativa na taxa de erros.

Como pudemos ver nos resultados de desempenho com pacotes curtos na Fig. 2, o desempenho de combinação de pacotes é limitado pela taxa de erros no cabeçalho MAC, que não pode ser melhorado através da combinação. Na Fig. 4 mostramos os ganhos de vazão que podem ser alcançados quando combinação de pacotes ideal é realizada, ou seja, supondo detecção perfeita de cabeçalho, e estes ganhos são bem significativos. Isto quer dizer que se o cabeçalho MAC for transmitido com maior confiabilidade, por exemplo empregando esquemas de modulação e codificação mais robustos para o cabeçalho que para os dados, podemos atingir ganhos substanciais de desempenho, particularmente em níveis baixos de RSR. Isto requer entretanto modificações na norma. Note que as curvas de vazão com combinação de pacotes rapidamente se aproximam de zero para baixos níveis de RSR, mas somente porque o número de retransmissões é limitado a dez. Se infinitas repetições fossem permitidas, as curvas tenderiam assintoticamente para zero com níveis decrescentes de RSR. 


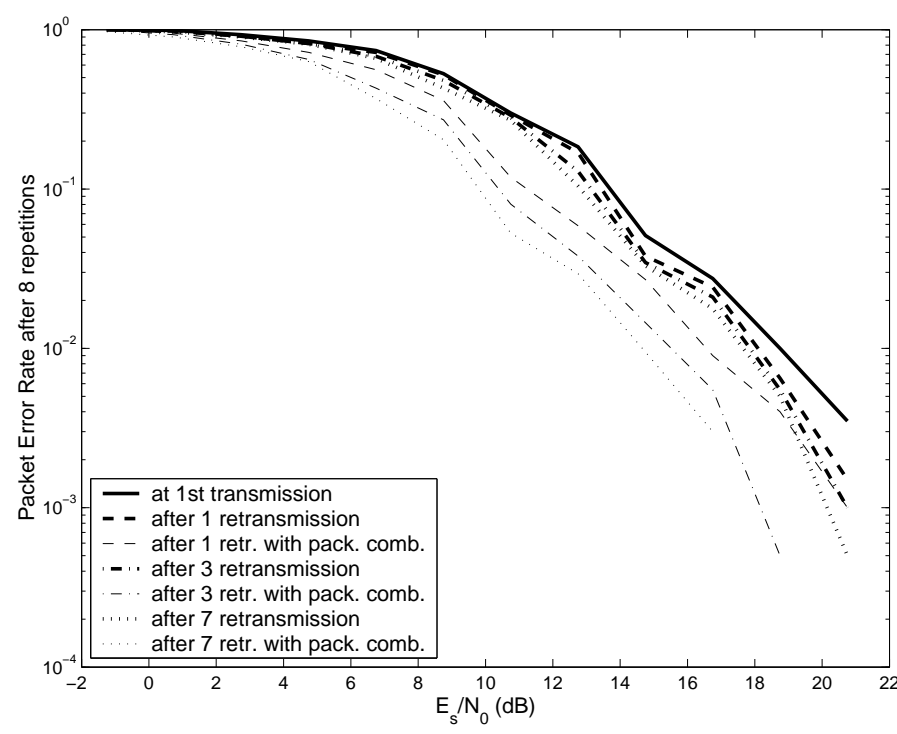

Fig. 3. Taxa de erro de pacotes com diferentes números de retransmissões

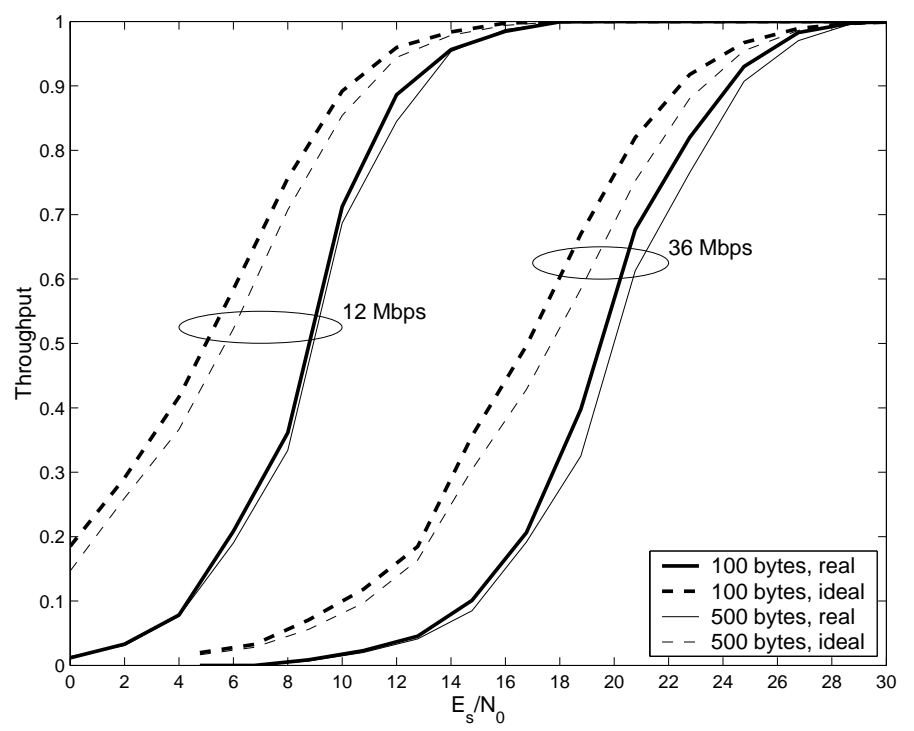

Fig. 4. Vazão com combinação de pacotes ideal e realística

\section{CONCLUSÕES}

Vimos neste artigo que o uso de HARQ Tipo I é viável em sistemas IEEE 802.11a, mesmo sem haver suporte para isto na norma, e que ganhos significativos podem ser alcançados com combinação e pacotes. Sugerimos também uma modificação na norma vigente, que permitiria uma realização maior dos ganhos de HARQ, sem implicar numa sinalização do uso ou não de HARQ. Outros aspectos foram idealizados e merecem mais estudos, como por exemplo o efeito da estimação de canal e sincronização no desempenho da combinação de pacotes.

\section{REFERÊNCIAS}

[1] IEEE Std 802.11a-1999, ” Part 11: Wireless LAN Medium Access Control (MAC) and Physical Layer (PHY) specifications - High-speed Physical Layer in the $5 \mathrm{GHz}$ Band"

[2] IEEE Std 802.11g, " Part 11: Wireless LAN Medium Access Control (MAC) and Physical Layer (PHY) specifications - "
[3] J.-F. Cheng, "Coding Performance of Hybrid ARQ Schemes", IEEE Trans. Commun., Vol. 54, No. 6, pp. 1017-1029, jun. 2006

[4] R. Van Nee, G. Awater, M. Morikura, H. Takanashi, M. Webster, e W. Halford, "New high-rate wireless LAN standards,", IEEE Commun. Mag., vol. 37, no. 12, pp. 82-88, dez. 1999.

[5] P. S. Sindhu, "Retransmission error control with memory," IEEE Trans. Commun., vol. COM-25, no. 5, pp. 473-479, maio 1977.

[6] D. Chase, "Code combining - a maximum-likelihood decoding approach for combining an arbitrary number of noisy packets,"IEEE Trans. Commun., vol. COM-33, no. 5, pp. 385-393, maio 1985.

[7] S. Kallel e D. Haccoun, "Sequential decoding with ARQ and code combining: A robust hybrid FEC/ARQ system,'IEEE Trans. Commun., vol. 36, no. 7, pp. 773-780, jul. 1988.

[8] F. Adachi, S. Ito, e K. Ohno, "Performance analysis of a time diversity ARQ in land mobile radio," IEEE Trans. Commun., vol. 37, no. 2, pp. 177-183, fev. 1989.

[9] B. A. Harvey e S. B. Wicker, "Packet combining systems based on the Viterbi decoder," IEEE Trans. Commun., vol. 42, nos. 2/3/4, pp. 15441557, fev./mar./abr. 1994.

[10] K. R. Narayanan e G. L. Stuber, "A novel ARQ technique using the turbo coding principle," IEEE Commun. Lett., vol. 1, no. 2, pp. 49-51, mar. 1997.

[11] E. C. Strinati, S. Simoens e J. Boutros, " Performance evaluation of some hybrid HARQ schemes in IEEE 802.11a networks, " Proc. VTC-Spring, Jeju, Coréia do Sul, apr. 2003, pp. 2735-2739

[12] L. Cai, Y. Wan, P. Song e L. Gui, "Improved HARQ scheme using channel quality feedback for OFDM systems," Proc. VTC-Spring, Milan, Itália, maio 2004, pp. 2735-2739

[13] "Criteria for comparison," Tech. Rep. EP BRAN\#9, 30701F, ETSI, Sophia Antipolis, France, jul. 1998. 